\title{
原位包覆纳米碳提升钴锰氧化物材料储锌性能
}

\author{
何锦俊张昊喆刘晓庆* 卢锡洪* \\ (中山大学化学学院 生物无机与合成化学教育部重点实验室 广州 510275)
}

\begin{abstract}
摘要 钴锰氧化物 $(\mathrm{CMO})$ 具有安全无毒、价格便宜、活性位点多等优点, 是极具潜力的锌离子电池正极材料. 目前, 充 放电过程中电极材料溶解造成的结构坞塌成为限制其发展的瓶颈问题. 本工作提出了一种原位碳包覆的方法来缓解 $\mathrm{CMO}$ 的溶解问题, 在提高材料容量的基础上成功地延长了其循环寿命. 通过简单的一步水热方法, 成功制备了纳米碳 $(\mathrm{nC})$ 包覆的钴锰氧化物 $(\mathrm{CMO} @ \mathrm{C})$ 材料, $\mathrm{X}$ 射线衍射 $(\mathrm{XRD}) 、 \mathrm{X}$ 射线光电子能谱 $(\mathrm{XPS}) 、$ 拉曼光谱(Raman)等表征证明, $\mathrm{nC}$ 包覆层的引入并没有改变 $\mathrm{CMO}$ 材料的基本组分结构. 而且, 相较于 $\mathrm{CMO}, \mathrm{CMO} @ \mathrm{C}$ 的电荷转移阻力更小, 离子扩散更 快, 表现出更优异的电化学性能. 在 $0.5 \mathrm{~A} \cdot \mathrm{g}^{-1}$ 的电流密度下, $\mathrm{CMO} @ \mathrm{C}$ 显示出 $271.9 \mathrm{mAh} \cdot \mathrm{g}^{-1}$ 的比容量, 且经过 1000 圈 循环后, 材料的比容量没有发生衰减, 性能大大优于 CMO 对比样品 (103.7 $\mathrm{mAh} \cdot \mathrm{g}^{-1} ; 130$ 圈失效). 由此可见, 纳米碳包 覆层可同时提升 CMO 的储锌容量和循环寿命. 本工作为双金属氧化物正极材料的优化改性提供了新的策略.

关键词 锌离子电池; 原位包覆; 碳; 锰基材料; 长寿命
\end{abstract}

\section{Enhancing $\mathrm{Zn}^{2+}$ Storage Capability of Cobalt Manganese Oxide by In-Situ Nanocarbon Coating}

\author{
He, Jinjun Zhang, Haozhe Liu, Xiaoqing* Lu, Xihong* \\ (MOE Key Laboratory of Bioinorganic and Synthetic Chemistry, School of Chemistry, Sun Yat-Sen University, \\ Guangzhou 510275, China)
}

\begin{abstract}
The cobalt manganese oxide (CMO), with the advantages of high safety, non-toxicity, easy to obtain, multiple active sites, holds great potential in constructions of $\mathrm{Zn}$-ion batteries (ZIBs). Yet, the dissolution of electrode materials into the electrolyte usually causes the structural collapse during repeated charge/discharge courses, which greatly limits the lifespan of ZIBs and thus restricts their further development. Herein, an in-situ coating method is developed to address this issue. Via a simple one-step hydrothermal method, a nanoscale carbon layer (denoted as $\mathrm{nC}$ ) is introduced onto the surface of

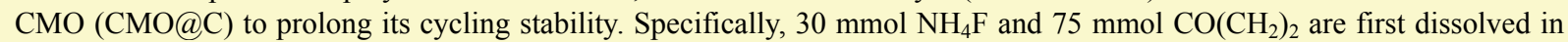
$100 \mathrm{~mL}$ deionized water. Then, $11.25 \mathrm{mmol} \mathrm{Mn}\left(\mathrm{CH}_{3} \mathrm{COO}\right)_{2}$ and $3.75 \mathrm{mmol} \mathrm{Co}\left(\mathrm{CH}_{3} \mathrm{COO}\right)_{2}$ are added and stirred until the solid completely dissolves. Finally, $0.5 \mathrm{~g}$ glucose is dissolved in the solution and stirred for $5 \mathrm{~min}$. The precursor solution is transferred into the $25 \mathrm{~mL}$ Teflon-lined stainless-steel autoclave and heated at $125{ }^{\circ} \mathrm{C}$ for $6 \mathrm{~h}$ in the oven. The as-obtained powder is washed three times by water and then dried at $60{ }^{\circ} \mathrm{C}$ overnight. The CMO@C sample is obtained after annealing the powder in air at $450{ }^{\circ} \mathrm{C}$ for $1 \mathrm{~h}$. The X-ray diffraction (XRD), X-ray photoelectron spectroscopy (XPS) and Raman spectra (Raman) characterizations demonstrate that the introduction of the $\mathrm{nC}$ coating layer does not alter the composition and structure of CMO. Moreover, taking advantages of the superior conductivity of the carbon coverage, the CMO@C possesses a smaller charge transfer resistance and higher $\mathrm{Zn}$ ion diffusion capability compared with the CMO counterpart. The quicker charge transfer and faster ion exchange characteristics are both beneficial to the electrochemical performance optimization, both for the capacity enlargement and for the lifespan extension. As a proof of concept, at the current density of $0.5 \mathrm{~A} \cdot \mathrm{g}^{-1}$, the CMO@C shows a high specific capacity of $271.9 \mathrm{mAh} \cdot \mathrm{g}^{-1}$ and no capacity loss is detected after 1000 cycle tests, which substantially outstrip those of the CMO (103.7 mAh•g ${ }^{-1}$ and 130 cycle lifespan). The work sheds light on the rational design of bimetal oxides as high-performance cathodes for ZIBs assembly.
\end{abstract}

Keywords Zn-ion battery; in-situ coating; carbon; manganese-base materials; long-term lifespan

\section{1 引言}

目前, 环境和化石能源储量的问题日益严峻, 人们 亟需开发、利用和储存各种可再生能源, 而建立大规模
的能源储存系统则是其中的一个关键步骤. 锂离子电池 (LIBs) 具有能量密度高、循环稳定性好等优点 ${ }^{[1]}$, 已经占 据世界电源市场主导地位几十年. 然而, 金属锂储量并

\footnotetext{
*E-mail: liuxiaoq5@mail.sysu.edu.cn; luxh6@mail.sysu.edu.cn

Received July 15, 2020; published September 11, 2020.

Supporting information for this article is available free of charge via the Internet at http://sioc-journal.cn

Project supported by the National Natural Science Foundation of China (Nos. 21822509, U1810110, 21802173) and Science and Technology Planning Project of Guangdong Province (No. 2018A050506028).

项目受国家自然科学基金(Nos. 21822509, U1810110, 21802173)和广东省科技计划项目(No. 2018A050506028)资助.
} 
不丰富, 加上近年来世界范围内对 LIBs 的需求大大增 加, 金属锂的价格飞速上涨, LIBs 的价格随之上升. 此 外, 由于 LIBs 使用的电解液为有机系电解液, 存在着一 定的安全隐患 ${ }^{[2-4]}$, 无法应用于规模化能源储存系统. 因 此, 研究人员在过去的十几年里一直致力于研发出利用 地球上丰度较高的金属元素作为载流体的离子电池来 替代 $\mathrm{LIBs}^{[5-7]}$. 由于金属锌具有无毒、储量丰富、高理论 容量 $\left(820 \mathrm{mAh} \cdot \mathrm{g}^{-1}\right)$ 、低氧化还原电势 $(0.762 \mathrm{~V} v \mathrm{vs}$. SHE) 等优点 ${ }^{[8]}$, 锌离子电池(ZIBs) 被认为是最有应用潜力的 离子电池之一. 且水系 ZIBs 使用不可燃的水系电解液, 安全性较好, 非常适用于大规模化的能量储存系统. 尽 管 ZIBs 具有巨大的应用前景, 但正极材料较差的电化 学稳定性严重制约了它的发展.

金属氧化物是 ZIBs 常用的一类正极材料 ${ }^{[9-11]}$. 相比 于单金属氧化物, 双金属氧化物中的金属离子通过 $\mathrm{M}$ $\mathrm{O}-\mathrm{M}^{\prime}\left(\mathrm{M} 、 \mathrm{M}^{\prime}\right.$ 为不同的金属离子)键连接, 能够提供更 多的活性位点, 得到更高的比容量. 因此, 人们发展了 一系列金属离子掺杂策略来构筑双金属氧化物以提高 储锌性能. 例如, Sada 等 ${ }^{[12]}$ 在 $\alpha-\mathrm{MnO}_{2}$ 中引入 $\mathrm{K}^{+}$离子制 备出 $\mathrm{K}_{1.33} \mathrm{Mn}_{8} \mathrm{O}_{16}$, 由于 $\mathrm{MnO}_{2}$ 中的 $\mathrm{Mn}^{4+}$ 与 $\mathrm{K}^{+}$产生了协 同效应，材料在 $\mathrm{C} / 10$ 下的比容量高达 $312 \mathrm{mAh} \cdot \mathrm{g}^{-1}$; 而 Bai 等 ${ }^{[13]}$ 通过溶胶凝胶法制备出 $\mathrm{Na}^{+}$掺杂的 $\mathrm{Na}_{0.44} \mathrm{MnO}_{2}$, 该材料在 $100 \mathrm{~mA} \cdot \mathrm{g}^{-1}$ 的电流密度下循环 150 圈后还显 示 $340 \mathrm{mAh} \cdot \mathrm{g}^{-1}$ 的比容量; 我们课题组 ${ }^{[14]}$ 也通过在 $\mathrm{MnO}_{2}$ 层间插入 $\mathrm{La}^{3+}$ 离子, 成功将其层间距从 $0.69 \mathrm{~nm}$ 扩 大到 $0.76 \mathrm{~nm}$ 以储存更多的锌离子, $\mathrm{La}^{3+}$ 插层的 $\mathrm{MnO}_{2}$ 较 对比样品表现出更高的倍率性能和比容量. 但是, 与单 金属氧化物类似 ${ }^{[15-17]}$, 双金属氧化物 ${ }^{[18-20]}$ 同样面临着充 放电过程中材料溶解的问题, 组装的 ZIBs 的循环寿命 一般较差.

在本工作中, 我们基于丰度较高的钴、锰元素, 通
过简单的一步水热法，制备了安全无毒、不易溶于水的 钴锰双金属氧化物 $(\mathrm{CMO})$ 作为 ZIBs 正极材料 ${ }^{[21-23]}$. 在 $\mathrm{CMO}$ 中，钴和锰都属于过渡金属，具有多种价态，能在 充放电过程中转移更多电子, 且其结构易于调控, 可调 控制备出具有多活性位点的结构，使其能储存更多的锌 离子, 从而能获得更高的比容量. 但其半导体的特性仍 然限制了其容量 ${ }^{[24,25]}$, 而且, 它的循环稳定性也比较差. 为了解决这些问题，我们提出在 $\mathrm{CMO}$ 表面原位包覆一 层纳米级厚度的碳材料 $(\mathrm{nC})$. 一方面, $\mathrm{nC}$ 包覆层具有高 导电特性, 可在不改变 $\mathrm{CMO}$ 组分结构的基础上, 有效 促进材料内部的离子传输和电荷转移, 增加其容量; 另 一方面, $\mathrm{nC}$ 包覆层可作为缓冲层抑制充放电过程中活性 物质 $\mathrm{CMO}$ 的溶解, 提升其寿命. 因此, 与 $\mathrm{CMO}$ 对比样 品相比较, $\mathrm{nC}$ 包覆的 CMO (CMO@C) 表现出更高的容 量和更好的循环稳定性. 在 $0.5 \mathrm{~A} \cdot \mathrm{g}^{-1}$ 的电流密度下, $\mathrm{CMO}$ 和 CMO@C 的比容量分别为 103.7 和 $271.9 \mathrm{mAh}$ • $\mathrm{g}^{-1}$; 且在 20 倍电流密度下 $\left(10 \mathrm{~A} \cdot \mathrm{g}^{-1}\right), \mathrm{CMO} @ \mathrm{C}$ 还有 $47.9 \%$ 的容量保持率 $\left(130.3 \mathrm{mAh} \cdot \mathrm{g}^{-1}\right)$, 而 $\mathrm{CMO}$ 仅为 $37.9 \%$. 此外, 在 $6 \mathrm{~A} \cdot \mathrm{g}^{-1}$ 电流密度下, $\mathrm{CMO} @ \mathrm{C}$ 经过 1000 圈循环测试后, 容量并没有发生衰减. 综上, $\mathrm{nC}$ 包 覆层可同时提高 $\mathrm{CMO}$ 材料的容量和电化学稳定性.

\section{2 结果与讨论}

通过简单的一步水热反应实现了 $\mathrm{CMO}$ 粉末的成功 制备(参见实验部分). 为了实现 $\mathrm{CMO}$ 的原位碳包覆, 在 不改变水热反应条件的前提下, 往前驱体液中添加葡萄 糖作为碳源. 水热合成后的样品在 $450{ }^{\circ} \mathrm{C} \mathrm{Ar}$ 气氛下煅 烧 $1 \mathrm{~h}$, 即得 $\mathrm{CMO} @ \mathrm{C}$ 样品. 我们对制备出的 CMO 与 $\mathrm{CMO} @ \mathrm{C}$ 样品进行了材料结构表征对比. 图 $1(\mathrm{a}, \mathrm{b})$ 中扫 描电镜图(SEM)表明, 经过煅烧后, $\mathrm{CMO}$ 的表面较为平 a

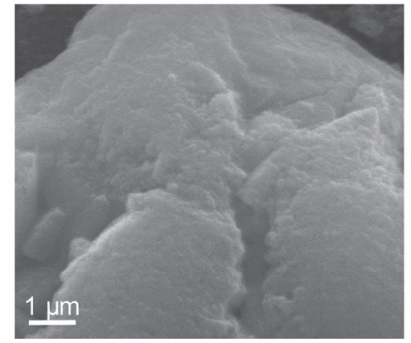

d

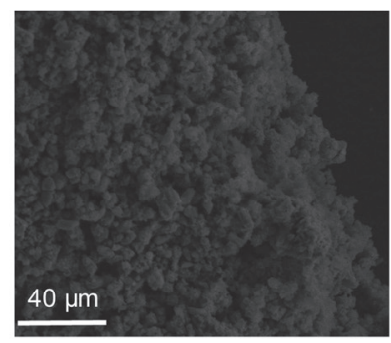

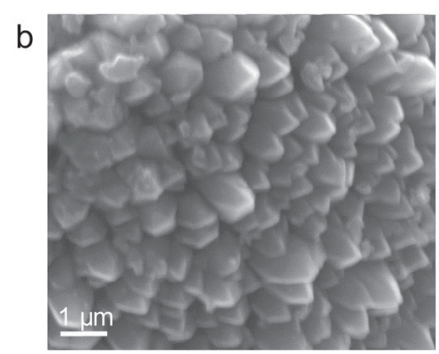
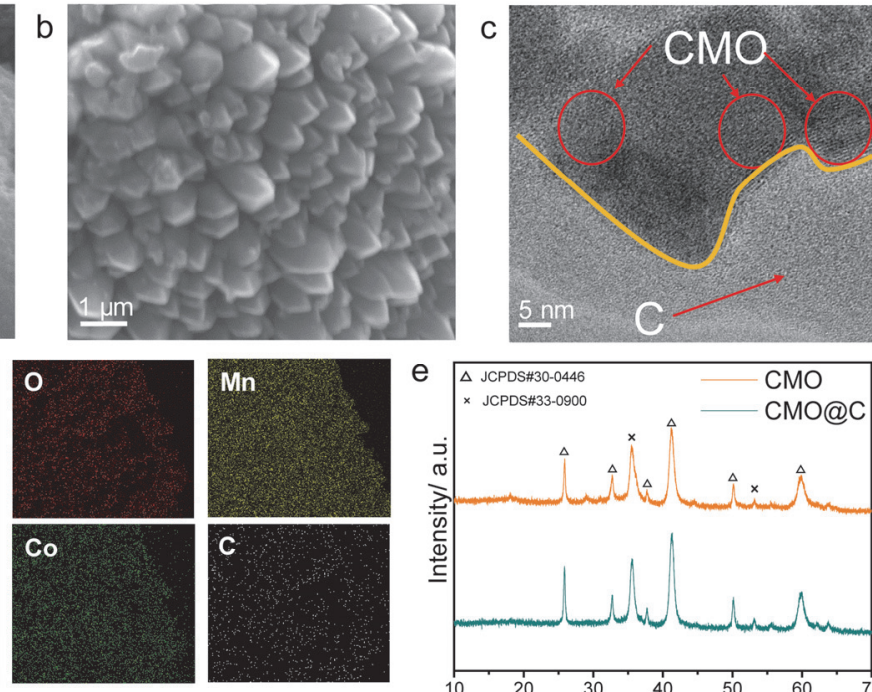

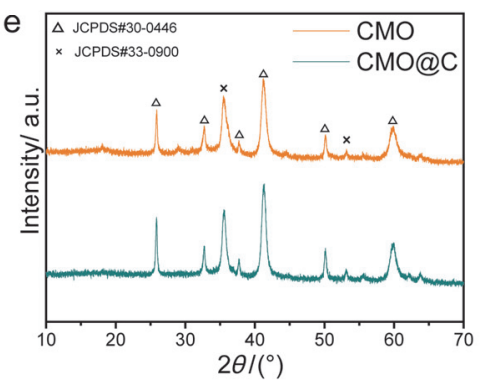

图 1 (a) $\mathrm{CMO}$ 与(b) $\mathrm{CMO} @ \mathrm{C}$ 的 SEM 图; $\mathrm{CMO} @ \mathrm{C}$ 的(c) TEM 与(d) 能谱图; (e) $\mathrm{CMO}$ 与 $\mathrm{CMO} @ \mathrm{C}$ 的 XRD 图

Figure 1 SEM images of (a) CMO and (b) CMO@C; (c) TEM and (d) EDS images of CMO@C; (e) XRD spectra of CMO and CMO@C 
整, 而 $\mathrm{nC}$ 包覆的 $\mathrm{CMO}$ 转变为菱形堆积结构, 形貌更为 规整, 这可能是由于 $\mathrm{nC}$ 保护层的存在抑制了 $\mathrm{CMO}$ 在高 温条件下的熔融和坡塌. 图 S1 与图 1(c)分别为 $\mathrm{CMO}$ 与 $\mathrm{CMO} @ \mathrm{C}$ 的透射电镜(TEM)图. 对于 $\mathrm{CMO} @ \mathrm{C}$ 样品, 有 晶格条纹的部分为 $\mathrm{CMO}$ 材料, 而无晶格条纹的部分则 为无定型的 $\mathrm{nC}$ 包覆层. 从图中可发现, 前驱体溶液中 葡萄糖碳源的引入，可以成功实现 $\mathrm{CMO}$ 表面无定型 $\mathrm{nC}$ 的原位包覆, 其厚度约为 $10 \sim 30 \mathrm{~nm}$. 纳米级碳包覆层 的存在有望在增强 $\mathrm{CMO}$ 导电性的同时稳定其结构, 从 而赋予 $\mathrm{CMO} @ \mathrm{C}$ 更好的电化学性能. 图 1(d)为 $\mathrm{CMO} @ \mathrm{C}$ 的能谱图, 结果显示, $\mathrm{Co} 、 \mathrm{Mn} 、 \mathrm{O} 、 \mathrm{C}$ 元素均匀分布在 $\mathrm{CMO} @ \mathrm{C}$ 样品中, 说明制备的 $\mathrm{CMO} @ \mathrm{C}$ 较为均一. 如图 $1(\mathrm{e})$, 为了研究材料的晶相结构, 我们对材料进行了 XRD 表征. 通过对比发现, CMO@C 的 XRD 谱图与 $\mathrm{CMO}$ 的一致, 说明通过添加葡萄糖进行水热反应, 只 是在 $\mathrm{CMO}$ 表面包覆了一层 $\mathrm{nC}$, 并没有改变 $\mathrm{CMO}$ 的晶 相结构. 经过与标准卡片的对比, 发现 CMO 与 $\mathrm{CMO} @ \mathrm{C}$ 的主体晶相为 $\mathrm{Co}_{2} \mathrm{Mn}_{3} \mathrm{O}_{8}$ (JCPDS\#30-0446) ${ }^{[26]}$, 除了 $\mathrm{Co}_{2} \mathrm{Mn}_{3} \mathrm{O}_{8}$, 样品中还含有部分 $\mathrm{Mn}_{2} \mathrm{O}_{3}$ (JCPDS\#33-0900), 这可能是在合成过程中, $\mathrm{Mn}^{2+}$ 未完 全发生反应残留剩下的.

为了探究 $\mathrm{nC}$ 包覆后, 材料组分构成是否发生变化, 我们进行了 XPS、Raman 和热重分析(TG)等测试. 图 $2(\mathrm{a}, \mathrm{b})$ 与图 $\mathrm{S} 2$ 分别为 $\mathrm{CMO}$ 与 $\mathrm{CMO} @ \mathrm{C}$ 的 Co $2 \mathrm{p}_{3 / 2} 、 \mathrm{Mn}$ $2 \mathrm{p} 、 \mathrm{O} 1 \mathrm{~s}$ 和 $\mathrm{C} 1 \mathrm{~s}$ 轨道的 XPS 图. 图 2(a)中位于 780.1 与 $781.7 \mathrm{eV}$ 的峰分别对应 $\mathrm{Co}^{3+}$ 与 $\mathrm{Co}^{2+}$ 的吸收峰, 而位于 785.7 和 $787.6 \mathrm{eV}$ 的吸收峰则是 $\mathrm{Co}^{3+}$ 与 $\mathrm{Co}^{2+}$ 的伴峰 ${ }^{[27]}$. 图 2(b) Mn 2p 轨道的 XPS 中位于 $641.8 \mathrm{eV}$ 的是 $\mathrm{Mn} 2 \mathrm{p}_{3 / 2}$ 的峰, 位于 $653.4 \mathrm{eV}$ 的是 $\mathrm{Mn} 2 \mathrm{p}_{1 / 2}$ 的峰, 根据 $\mathrm{Mn} 2 \mathrm{p}_{3 / 2}$
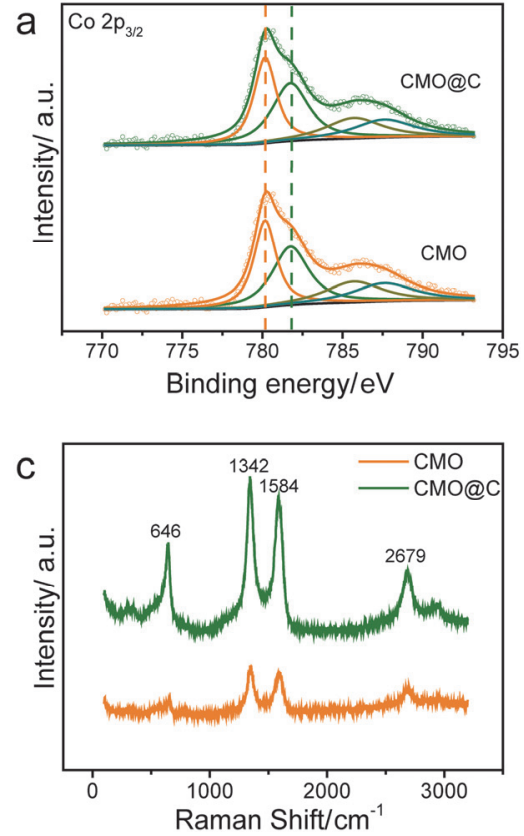

与 $\mathrm{Mn} 2 \mathrm{p}_{1 / 2}$ 的峰位差, $\mathrm{CMO}$ 中的 $\mathrm{Mn}$ 元素的价态为+3 价 ${ }^{[28]}$. 图 2(a,b)与图 S2 表明, Co、Mn、O 元素的 XPS 峰没有发生位移, 即这些元素的化学价态没有发生改 变, 进一步说明了 $\mathrm{nC}$ 包覆没有改变 $\mathrm{CMO}$ 材料的组分构 成. 图 2(c)为 $\mathrm{CMO}$ 与 $\mathrm{CMO} @ \mathrm{C}$ 的 Raman 光谱图, 位于 $646 \mathrm{~cm}^{-1}$ 的峰是属于 $\mathrm{CMO}$ 中 $\mathrm{Co}-\mathrm{O}$ 的信号峰 ${ }^{[29]}$, 而在 1342、1584 和 $2679 \mathrm{~cm}^{-1}$ 位置的峰则是材料中 $\mathrm{C}$ 的 $\mathrm{D}$ 峰、 $\mathrm{G}$ 峰和 $2 \mathrm{D}$ 峰 $^{[30]}$. 其中, CMO 中检测的 C 信号可能 来源于水热反应含碳助剂(如 $\mathrm{CO}\left(\mathrm{NH}_{2}\right)_{2}$ 等)殷烧后的残 留.

为了判断 $\mathrm{CMO} @ \mathrm{C}$ 材料中包覆 $\mathrm{nC}$ 的含量, 我们对 $\mathrm{CMO}$ 与 $\mathrm{CMO} @ \mathrm{C}$ 进行了空气中 $\mathrm{TG}$ 分析. 如图 2(d)所 示, $\mathrm{CMO}$ 样品在低于 $200{ }^{\circ} \mathrm{C}$ 的温度区间时, 失重约 $0.70 \%, \mathrm{CMO} @ \mathrm{C}$ 样品失重约 $0.57 \%$, 这是因为样品中含 有的少量水蒸发导致的; 在 $200 \sim 400{ }^{\circ} \mathrm{C}$ 间, 两个样品 的总质量都增加了, $\mathrm{CMO}$ 材料总质量增加到约 $101.77 \%, \mathrm{CMO} @ \mathrm{C}$ 样品则增加到 102.27\%, 这可归因于 样品中含有的低价态的过渡金属元素被完全氧化而导 致的总质量增加; 而在 $400 \sim 900{ }^{\circ} \mathrm{C}$ 间, 由于样品中含 有的 C 元素氧化 ${ }^{[31]}$ ，导致 CMO 样品质量减少到 $98.18 \%$, 而 CMO@C 样品的质量则减少到 96.28\%. 如前所述, $\mathrm{CMO}$ 样品中的 $\mathrm{C}$ 元素来源于合成过程中 $\mathrm{CO}\left(\mathrm{NH}_{2}\right)_{2}$ 等含 碳助剂残留, 而 $\mathrm{CMO} @ \mathrm{C}$ 样品中的 $\mathrm{C}$ 元素除了助剂残 留, 还来源于 $\mathrm{nC}$ 包覆层中的 $\mathrm{C}$ 元素, 故 $\mathrm{CMO} @ \mathrm{C}$ 样品 的含碳量减去 $\mathrm{CMO}$ 样品的含碳量则可得到 $\mathrm{CMO} @ \mathrm{C}$ 样 品中 $\mathrm{nC}$ 包覆层的含量. 经过计算, $\mathrm{CMO}$ 和 $\mathrm{CMO} @ \mathrm{C}$ 中 $\mathrm{C}$ 的质量分数分别约为 $3.59 \%$ 和 $5.99 \%$, 则计算得到通 过水热法在 $\mathrm{CMO}$ 表面包覆的 $\mathrm{nC}$ 包覆层的含量约为 $2.40 \%$
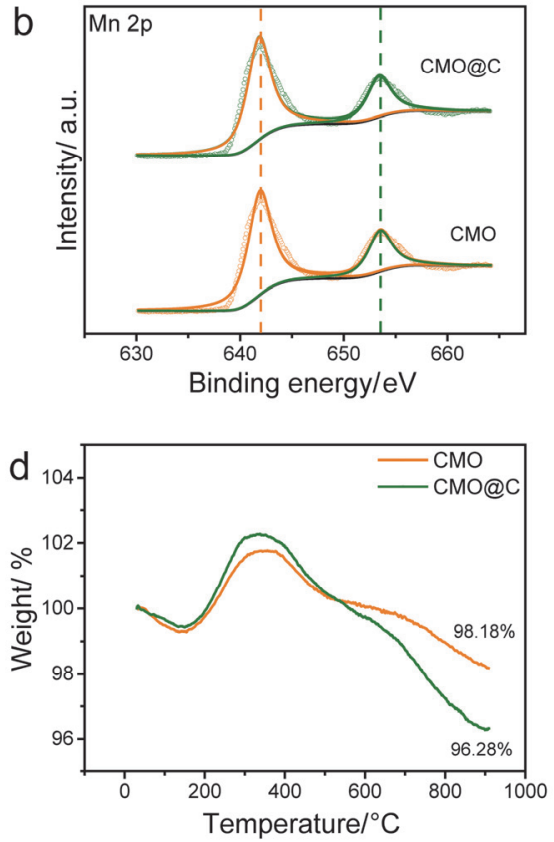

图 $2 \mathrm{CMO}$ 和 $\mathrm{CMO} @ \mathrm{C}$ 的(a) Co $2 \mathrm{p}$ 轨道与(b) Mn 2p 轨道的 XPS 图; (c) Raman 光谱图和(d) 热重分析图

Figure 2 XPS of (a) Co 2p and (b) Mn 2p; (c) Raman spectra and (d) TG analyzation of CMO and CMO@C 
为了探究 $\mathrm{CMO} @ \mathrm{C}$ 的结构优势与其电化学性能, 我们以负载 $\mathrm{CMO}$ 或 $\mathrm{CMO} @ \mathrm{C}$ 的碳纸为正极, 商业金属 锌片为负极, 使用含有 $1 \mathrm{~mol} \cdot \mathrm{L}^{-1} \mathrm{ZnCl}_{2}$ 和 $0.4 \mathrm{~mol} \cdot \mathrm{L}^{-1}$ $\mathrm{MnSO}_{4}$ 的水溶液作为电解液组装了 $\mathrm{CMO} / / \mathrm{Zn}$ 与 $\mathrm{CMO} @ \mathrm{C} / / \mathrm{Zn}$ 两种锌离子电池, 并分别测试了它们的电 化学性能. 为了比较两种材料的容量, 我们对 $\mathrm{CMO} / / \mathrm{Zn}$ 与 $\mathrm{CMO} @ \mathrm{C} / / \mathrm{Zn}$ 电池进行了恒定充放电(GCD)测试. 如 图 3(a,b)所示, 在相同电流密度下, $\mathrm{CMO} @ \mathrm{C} / / \mathrm{Zn}$ 电池 总是具有更长的放电平台, 显示出更高的比容量. 在

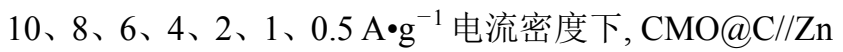
电池的比容量分别为 $130.3 、 135.5 、 148.1 、 170.7 、 213.0$ 、

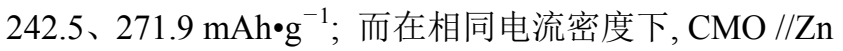
电池仅分别显示出 30.4、41.8、46.2、55.1、73.0、88.6、 $103.7 \mathrm{mAh} \cdot \mathrm{g}^{-1}$ 的比容量.

图 3(c) 与图 $S 3$ 为两种电池的倍率性能图, 可以直观 地看到, $\mathrm{CMO} @ \mathrm{C} / / \mathrm{Zn}$ 较 $\mathrm{CMO} / / \mathrm{Zn}$ 显示出更好的倍率性 能, 在 20 倍的电流密度下 $\left(10 \mathrm{~A} \cdot \mathrm{g}^{-1}\right)$ 还保持着 $47.9 \%$ 的 比容量，而相同条件下 $\mathrm{CMO}$ 的容量保持率仅为 $37.9 \%$. $\mathrm{CMO} @ \mathrm{C}$ 较高的比容量源于其更快的电化学动力学过 程, 即 $\mathrm{nC}$ 包覆层优异的导电性解决了 $\mathrm{CMO}$ 导电性差的 问题, 有效促进了锌离子的快速吸脱附. 为了研究材料 在电化学充放电过程中的稳定性, 我们在 $6 \mathrm{~A} \cdot \mathrm{g}^{-1}$ 的电 流密度下, 对材料进行了恒电流循环充放电测试. 从图 3(d)中可以看到, $\mathrm{CMO} / / \mathrm{Zn}$ 电池在经过 130 圈循环后即 不能继续充放电, 这可能是因为在反复的充放电过程 中, 裸露的 $\mathrm{CMO}$ 逐渐溶解到电解液中, 引起材料结构 坞塌. 而 $\mathrm{CMO} @ \mathrm{C} / / \mathrm{Zn}$ 在 1000 圈循环充放电测试后, 仍 然没有发生容量衰减. $\mathrm{CMO} @ \mathrm{C}$ 更长的循环寿命证明了
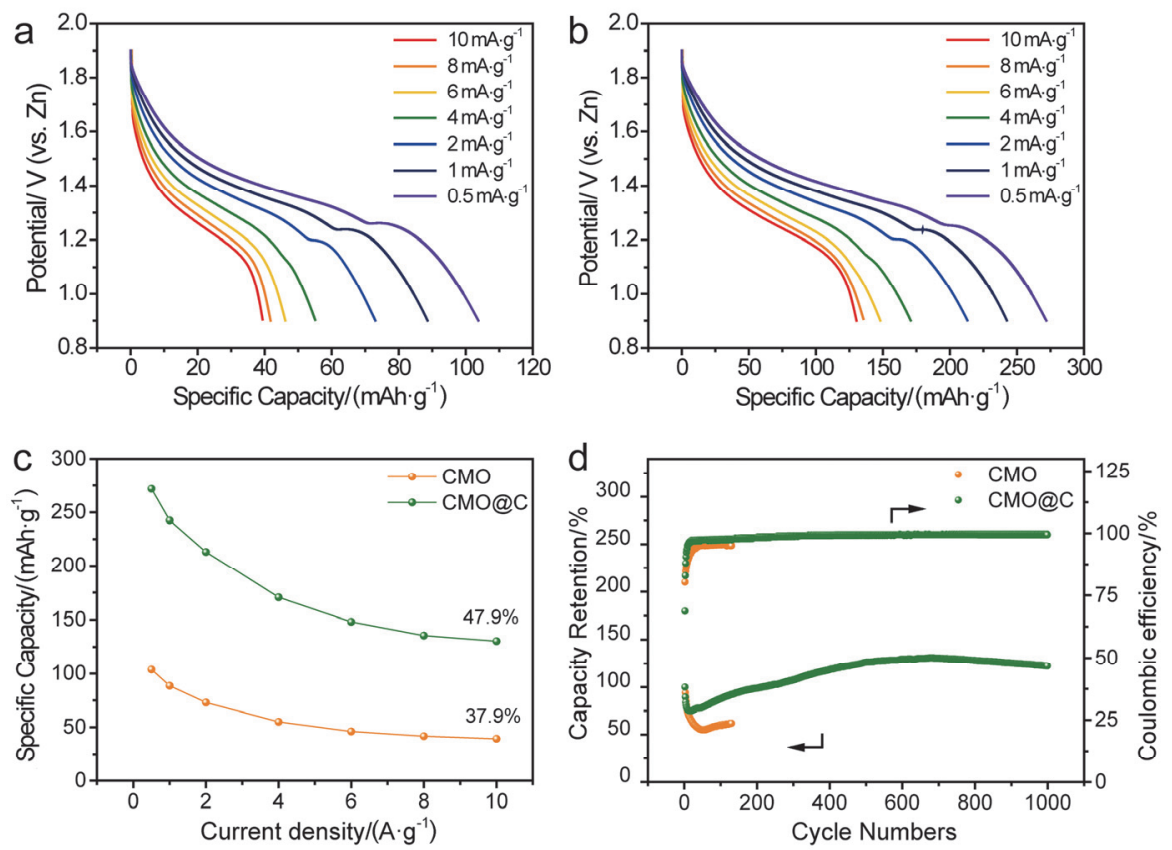

图 3 (a) $\mathrm{CMO} / / \mathrm{Zn}$ 和(b) $\mathrm{CMO} @ \mathrm{C} / / \mathrm{Zn}$ 电池在不同电流密度下的恒电流放电曲线; $\mathrm{CMO} / / \mathrm{Zn}$ 和 $\mathrm{CMO} @ \mathrm{C} / / \mathrm{Zn}$ 电池的(c)倍率性能图与(d)循环寿命图 Figure $3 \mathrm{GCD}$ curves at various current densities of (a) $\mathrm{CMO} / / \mathrm{Zn}$ and (b) $\mathrm{CMO} @ \mathrm{C} / / \mathrm{Zn}$ batteries; (c) rate performance and (d) long-term cycling performance of $\mathrm{CMO} / / \mathrm{Zn}$ and $\mathrm{CMO} @ \mathrm{C} / / \mathrm{Zn}$ batteries 

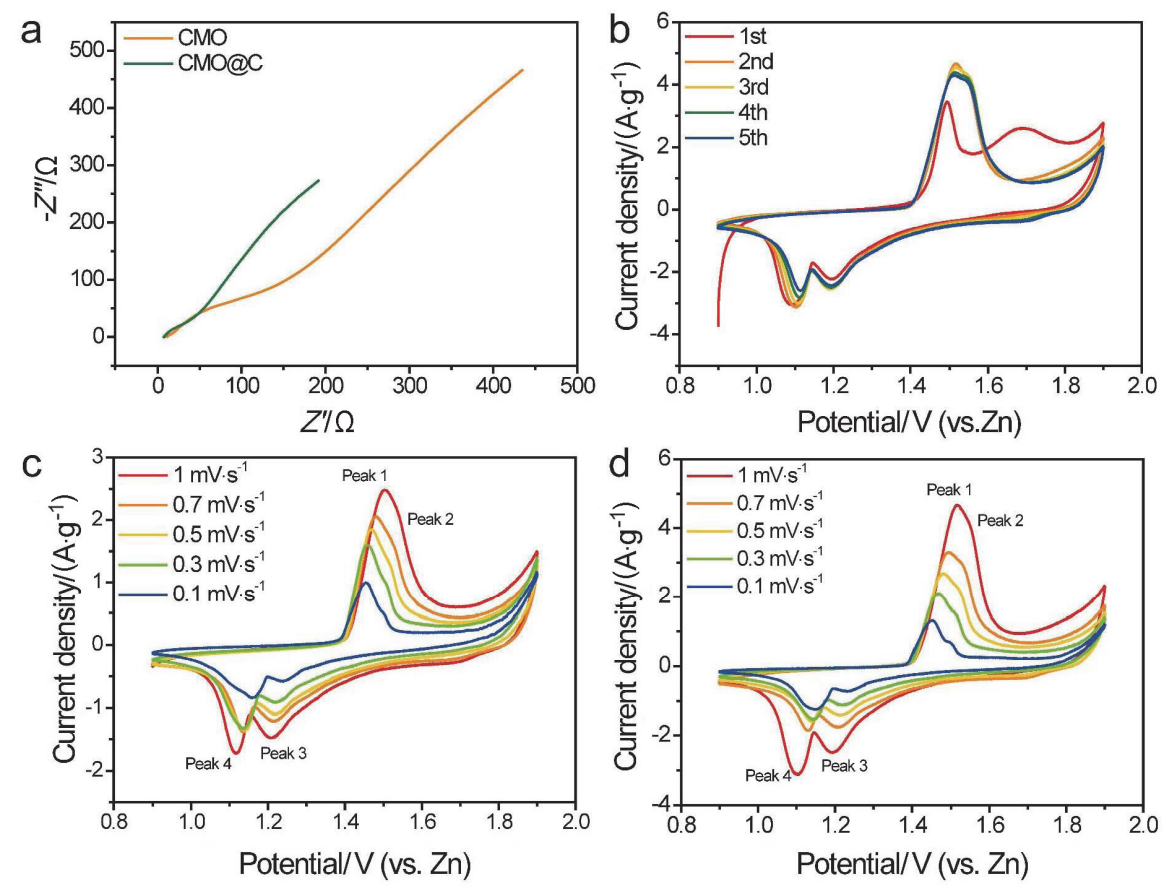

图 4 (a) $\mathrm{CMO} / / \mathrm{Zn}$ 与 $\mathrm{CMO} @ \mathrm{C} / / \mathrm{Zn}$ 电池在开路电位下的 $\mathrm{EIS}$ 图; (b) $\mathrm{CMO} @ \mathrm{C} / / \mathrm{Zn}$ 电池在 $1 \mathrm{mV} \cdot \mathrm{s}^{-1}$ 扫描速率下首 5 圈 $\mathrm{CV}$ 图; (c) $\mathrm{CMO} / / \mathrm{Zn}$ 与(d) $\mathrm{CMO} @ \mathrm{C} / / \mathrm{Zn}$ 电池在不同扫描速率下的 $\mathrm{CV}$ 曲线图

Figure 4 (a) EIS at open circuit potential of $\mathrm{CMO} / / \mathrm{Zn}$ and $\mathrm{CMO} @ \mathrm{C} / / \mathrm{Zn}$ batteries; (b) the first five cycles for $\mathrm{CV}$ curves of the $\mathrm{CMO} @ \mathrm{C} / / \mathrm{Zn}$ battery at the scan rate of $1 \mathrm{mV} \cdot \mathrm{s}^{-1}$; the $\mathrm{CV}$ curves of (c) $\mathrm{CMO} / / \mathrm{Zn}$ and (d) $\mathrm{CMO} @ \mathrm{C} / / \mathrm{Zn}$ batteries at various scan rates

$0.7 、 1.0 \mathrm{mV} \cdot \mathrm{s}^{-1}$ 扫描速率下的 $\mathrm{CV}$ 曲线图. 两个电池的 $\mathrm{CV}$ 曲线具有相似的氧化还原峰, 说明 $\mathrm{nC}$ 的包覆没有改 变 $\mathrm{CMO}$ 在电化学过程中的氧化还原反应过程. 另外, 无论在何种扫速条件下, $\mathrm{nC}$ 的包覆 $\mathrm{CMO} @ \mathrm{C}$ 总是比 $\mathrm{CMO}$ 表现出更高的电流密度和更大的曲线面积, 说明 $\mathrm{CMO} @ \mathrm{C}$ 具有更好的储锌能力, 这也与其表现出的高容 量相对应. 我们根据 CV 中峰电流与扫描速率的关系作 图(图 S5), 证明 $\mathrm{CMO} @ \mathrm{C} / / \mathrm{Zn}$ 电池具有更好的性能.

我们将CMO@C//Zn 电池与其他已报道的电池的能 量密度与功率密度做比较(图 5), 证实 $\mathrm{CMO} @ \mathrm{C} / / \mathrm{Zn}$ 电 池性能的优势. CMO@C//Zn 电池能获得最大的能量密

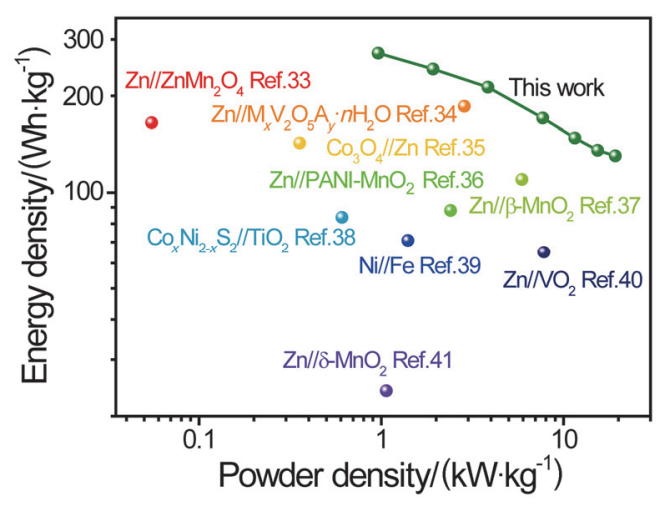

图 $5 \mathrm{CMO} @ \mathrm{C} / / \mathrm{Zn}$ 电池与其他已报道的电池的能量密度与功率密度 的对比图

Figure 5 Ragone plots comparing the energy densities and power densities values of $\mathrm{CMO} @ \mathrm{C} / / \mathrm{Zn}$ with those of other reported batteries
度有 $271.9 \mathrm{Wh} \cdot \mathrm{kg}^{-1}$, 且在 $19.22 \mathrm{~kW} \cdot \mathrm{kg}^{-1}$ 的功率密度下 显示出 $130.3 \mathrm{Wh} \cdot \mathrm{kg}^{-1}$ 的能量密度, 明显优于其他报道 的电池, 如 $\mathrm{Zn} / / \mathrm{ZnMn}_{2} \mathrm{O}_{4}$ 电池 ${ }^{[33]}, \mathrm{Zn} / / \mathrm{M}_{x} \mathrm{~V}_{2} \mathrm{O}_{5} \mathrm{~A}_{y} \cdot n \mathrm{H}_{2} \mathrm{O}$ 电 池 ${ }^{[34]}, \mathrm{Co}_{3} \mathrm{O}_{4} / / \mathrm{Zn}$ 电池 ${ }^{[35]}, \mathrm{Zn} / / \mathrm{PANI}$-intercalated $\mathrm{MnO}_{2}$ 电 池 ${ }^{[36]}, \mathrm{Zn} / / \beta-\mathrm{MnO}_{2}$ 电池 ${ }^{[37]}, \mathrm{Co}_{x} \mathrm{Ni}_{2-\mathrm{x}} \mathrm{S}_{2} / / \mathrm{TiO}_{2}$ 电池 ${ }^{[38]}$, $\mathrm{Ni} / / \mathrm{Fe}$ 电池 ${ }^{[39]}, \mathrm{Zn} / / \mathrm{VO}_{2}$ 电池 ${ }^{[40]}, \mathrm{Zn} / / \delta-\mathrm{MnO}_{2}$ 电池 ${ }^{[41]}$.

\section{3 结论}

通过原位水热合成法, 成功地在 $\mathrm{CMO}$ 材料表面包 覆了一层 $\mathrm{nC}$, 制备出高比容量、长寿命的水系锌离子电 池 CMO@C 材料. 通过 TEM 图表征，在 CMO 材料表面 包覆的 $\mathrm{C}$ 的厚度为纳米级别. 经过 XRD、XPS、Raman、 $\mathrm{CV}$ 等表征, 证明了 $\mathrm{nC}$ 包覆层不会改变 $\mathrm{CMO}$ 原有的组 分结构, 没有破坏其活性位点. 而由于 $\mathrm{C}$ 材料高电导 率、高稳定性等优点, $\mathrm{nC}$ 的包覆能让 $\mathrm{CMO}$ 材料拥有更 稳定的结构、更低的离子扩散阻力和电荷转移阻力、更 快的电化学动力学, 使得材料具有更大的比容量、更高 的倍率性能和更好的循环稳定性. 制备的水系 $\mathrm{CMO} @ \mathrm{C} / / \mathrm{Zn}$ 电池在 $0.5 \mathrm{~A} \cdot \mathrm{g}^{-1}$ 的电流密度下有 271.87 $\mathrm{mAh} \cdot \mathrm{g}^{-1}$ 的高比容量, 而在 20 倍的电流密度下, 还有着 $47.9 \%$ 的容量保持率. 此外, $\mathrm{CMO} @ \mathrm{C}$ 电极在 $6 \mathrm{~A} \cdot \mathrm{g}^{-1}$ 的 电流密度下循环 1000 圈, 容量没有发生衰减. 可见, $\mathrm{nC}$ 包覆可同时提升 CMO 的容量和循环寿命. 这项研究可 作为优化具有较差电化学性能的过渡金属氧化物材料 的参考, 为制备出有着更优异电化学性能的锌离子电池 
正极材料提供了方向.

\section{4 实验部分}

\section{$4.1 \mathrm{CMO} @ \mathrm{C}$ 的制备}

先将 $30 \mathrm{mmol}$ 的 $\mathrm{NH}_{4} \mathrm{~F}$ 和 $75 \mathrm{mmol}$ 的 $\mathrm{CO}\left(\mathrm{NH}_{2}\right)_{2}$ 溶 解于 $100 \mathrm{~mL}$ 去离子水, 再向溶液中加入 $11.25 \mathrm{mmol}$ 的 $\mathrm{Mn}\left(\mathrm{CH}_{3} \mathrm{COO}\right)_{2}$ 和 $3.75 \mathrm{mmol}$ 的 $\mathrm{Co}\left(\mathrm{CH}_{3} \mathrm{COO}\right)_{2}$ 搅拌至溶 解完全, 最后加入 $0.5 \mathrm{~g}$ 葡萄糖, 继续搅拌 $5 \mathrm{~min}$ 至均匀. 将配置好的溶液倒入 $25 \mathrm{~mL}$ 的反应釜中, 在 $125{ }^{\circ} \mathrm{C}$ 的温 度下水热反应 $6 \mathrm{~h}$. 待反应完成冷却至室温后, 刮取反 应釜内胆壁上粉末样品进行抽滤, 并用去离子水洗涤三 次, 将清洗后的粉末样品放至 $60{ }^{\circ} \mathrm{C}$ 烘箱中进行过夜干 燥. 干燥后的样品放置于 $\mathrm{Ar}$ 气氛下升温至 $450{ }^{\circ} \mathrm{C}$ 炦烧 1 $\mathrm{h}$, 得到 $\mathrm{CMO} @ \mathrm{C}$ 样品.

\section{2 $\mathrm{CMO}$ 的制备}

$\mathrm{CMO}$ 样品的制备方法与 $\mathrm{CMO} @ \mathrm{C}$ 的相同, 只需在 水热反应过程中, 不添加葡萄糖作为包覆 $\mathrm{nC}$ 的碳源.

\section{3 电极的制备}

$\mathrm{CMO}$ 电极和 $\mathrm{CMO} @ \mathrm{C}$ 电极都是通过将材料与聚偏 二氟乙烯、乙炔黑以 $8: 1: 1$ 的质量比混合, 并溶于 $1-$ 甲基-2-吡咯烷酮. 搅拌 $4 \mathrm{~h}$ 后将混合均匀的浆料涂抹于 碳纸上, 其后将样品电极放到 $60{ }^{\circ} \mathrm{C}$ 的真空干燥箱内干 燥过夜. 所使用的工作电极面积约为 $0.5 \mathrm{~cm}^{2}$, 碳纸上负 载的 $\mathrm{CMO}$ 或 $\mathrm{CMO} @ \mathrm{C}$ 的质量约为 $1 \mathrm{mg}$.

\section{4 材料表征及测试}

采用能谱-场发射扫描电镜(FE-SEM, JSM-6330F)、 透射电镜(TEM, FEI Tecnai G2 F30)和 X 射线衍射(XRD, DMAX $2200 \mathrm{VPC}$, RIGAKU)表征材料的形貌和晶体结 构; 拉曼光谱(Raman spectroscopy Renishaw inVia)、X 射线光电子能谱(XPS, NEXSA, Thermo VG)则用于表征 材料的成分与结构; 热重分析(TG, TG-209/Vector-22)用 于表征材料内 $\mathrm{nC}$ 的含量; 电感耦合等离子体发射光谱 (ICP-OES, Optima 8300, PerkinElmer)用于测量电池循环 后锰离子含量; 使用电化学工作站(CHI 760E)对材料进 行 CV、GCD、EIS 等电化学测试; 以商业锌片作为对电 极、含有 $1 \mathrm{~mol} \cdot \mathrm{L}^{-1} \mathrm{ZnCl}_{2}$ 与 $0.4 \mathrm{~mol} \cdot \mathrm{L}^{-1} \mathrm{MnSO}_{4}$ 的水溶 液作为电解液组装成两电极系统进行测试.

\section{References}

[1] Tian, C.; Tian, J.; Chen, F.; Tong, L; Gao, S.; Xu, C.; Wang, Z. J. Chongqing University Tech. (Natural Science) 2018, 10, 34 (in Chinese). (田崔钧, 田君, 陈芬, 佟蕾, 高申, 徐春常, 王子冬, 重庆 理工大学学报 (自然科学) , 2018, 10, 34.)

[2] Tang, G.; Mao, K.; Zhang, J.; Lyu, P.; Cheng, X.; Wu, Q.; Yang, L.; Wang, X.; Hu, Z. Acta Chim. Sinica 2020, 78, 444 (in Chinese). (汤 功奥, 毛鲲, 张静, 吕品, 程雪怡, 吴强, 杨立军, 王喜章, 胡征, 化学学报, 2020, 78, 444.)

[3] Wang, X.; Li, Y.; Du, L.; Gao, F.; Wu, Q.; Yang, L.; Chen, Q.; Wang, X.; Hu, Z. Acta Chim. Sinica 2018, 76, 627 (in Chinese). (主啸, 李 有涁, 杜玲玉, 高福杰, 吴强, 杨立军, 陈强, 王喜章, 胡征, 化
学学报, 2018, 76, 627.)

[4] Wang, L.; Zhao, D.; Liu, X.; Yu, P.; Fu, H. Acta Chim. Sinica 2017 75,231 (in Chinese). (王蕾, 赵冬冬, 刘旭, 于鹏, 付宏刚, 化学 学报, 2017, 75, 231.)

[5] Bauer, A.; Song, J.; Vail, S.; Pan, W.; Barker, J.; Lu, Y. Adv. Energy Mater. 2018, 8, 1702869 .

[6] Zhang, L.; Zhang, B.; Wang, C.; Dou, Y.; Zhang, Q.; Liu, Y.; Gao, H.; Al-Mamun, M.; Pang, W.; Guo, Z.; Dou, S.; Liu, H.; Nano Energy 2019, 60, 432.

[7] Zeng, Y.; Zhang, X.; Qin, R.; Liu, X.; Fang, P.; Zheng, D.; Tong, Y.; Lu, X. Adv. Mater. 2019, 31, 1903675.

[8] He, J.; Liu, X.; Zhang, H.; Yang, Z.; Shi, X.; Liu, Q.; Lu, X. ChemSusChem 2020, 13, 1568.

[9] Tang, B.; Shan, L.; Liang, S.; Zhou, J. Energy Envir. Sci. 2019, 12, 3288.

[10] Alfaruqi, M.; Mathew, V.; Gim, J.; Kim, S.; Song, J.; Baboo, J.; Choi, S.; Kim, J. Chem. Mater. 2015, 27, 3609.

[11] He, X.; Zhang, H.; Zhao, X.; Zhang, P.; Chen, M.; Zheng, Z.; Han, Z.; Zhu, T.; Tong, Y.; Lu, X. Adv. Sci. 2019, 6, 1900151

[12] Sada, K.; Senthilkumar, B.; Barpanda, P. J. Mater. Chem. A 2019, 7, 23981.

[13] Bai, S.; Song, J.; Wen, Y.; Cheng, J.; Cao, G.; Yang, Y.; Li, D. RSC Adv. 2016, 6, 40793.

[14] Zhang, H.; Liu, Q.; Wang, J.; Chen, K.; Xue, D.; Liu, J.; Lu, X. J. Mater. Chem. A 2019, 7, 22079.

[15] Wu, C.; Gu, S.; Zhang,Q.; Bai, Y.; Li, M.; Yuan, Y.; Wang, H.; Liu, X.; Yuan, Y.; Zhu, N.; Wu, F.; Li, H.; Gu, L.; Lu, J. Nat. Commun. 2019, 10, 73 .

[16] Xiong, T.; Yu, Z.; Wu, H.; Du, Y.; Xie, Q.; Chen, J.; Zhang, Y.; Pennycook, S.; Lee, W.; Xue, J. Adv. Energy Mater. 2019, 9, 1803815.

[17] Pan, H.; Shao, Y.; Yan, P.; Cheng, Y.; Han, K.; Nie, Z.; Wang, C.; Yang, J.; Li, X.; Bhattacharya, P.; Mueller, K.; Liu, J. Nat. Energy 2016, $1,16039$.

[18] Soundharrajan, V.; Sambandam, B.; Kim, S.; Mathew, V.; Jo, J.; Kim, S.; Lee, J.; Islam, S.; Kim, K.; Sun, Y.; Kim, J. ACS Energy Lett. 2018, 3, 1998.

[19] Zhang, N.; Cheng, F.; Liu, Y.; Zhao, Q.; Lei, K.; Chen, C.; Liu, X.; Chen, J. J. Am. Chem. Soc. 2016, 138, 12894.

[20] Zhang, H.; Wang, J.; Liu, Q.; He, W.; Lai, Z.; Zhang, X.; Yu, M.; Tong, Y.; Lu, X. Energy Storage Mater. 2019, 21, 154.

[21] Zhou, X.; Li, X.; Liao, B. J. Chongqing University Tech. (Natural Science) 2018, 7, 124 (in Chinese). (周小燕, 李小平, 廖本洪, 重 庆理工大学学报 (自然科学), 2018, 7, 124.)

[22] Liu, L.; Qi, X.; Hu, Y.; Chen, L.; Huang, X. Acta Chim. Sinica 2017, 75,218 (in Chinese). (刘丽露, 戚兴国, 胡勇胜, 陈立泉, 黄学杰, 化学学报, 2017, 75, 218.)

[23] Zhou, Y.; Chen, T.; Zhang, J.; Liu, Y.; Ren, P. Chin. J. Chem. 2017, $35,1294$.

[24] Mo, X.; Liu, W.; Xie, J.; Luo, R.; Hu, S. J. Chongqing University Tech. (Natural Science) 2020, 5, 220 (in Chinese). (莫兴丹, 刘伟, 谢健, 罗嵘, 胡顺仁, 重庆理工大学学报 (自然科学), 2020, 5, 220.)

[25] Zheng, Z.; Wu, Z.; Xiang, W.; Guo, X. Acta Chim. Sinica 2017, 75, 501 (in Chinese). (郑卓, 吴振国, 向伟, 郭孝东, 化学学报, 2017, 75,501 .)

[26] Zhu, H.; Gu, L.; Yu, D.; Sun, Y.; Wan, M.; Zhang, M.; Wang, L.; Wang, L.; Wu, W.; Yao, J.; Du, M.; Guo, S. Energy Envir. Sci. 2017, 10,321 .

[27] Lu, Y.; Wang, J.; Zeng, S.; Zhou, L.; Xu, W.; Zheng, D.; Liu, J.; Zeng, Y.; Lu, X. J. Mater. Chem. A 2019, 7, 21678.

[28] Sumi, V. S.; Elias, L.; Shibli, S. M. A. Int. J. Hydrogen Energy 2020 , 45,12360 .

[29] Zhao, Z.; Lin, J.; Wang, G.; Muhammad, T. AIChE J. 2015, 61, 239.

[30] Zeng, Y.; Lin, Z.; Wang, Z.; Wu, M.; Tong, Y.; Lu, X. Adv. Mater. 2018, 30, 1707290 .

[31] Wang, C.; Zeng, Y.; Xiao, X.; Wu, S.; Zhong, G.; Xu, K.; Wei, Z.; $\mathrm{Su}, \mathrm{W}$.; Lu, X. J. Energy Chem. 2020, 43, 182.

[32] Zhang, H.; Liu, Q.; Fang, Y.; Teng, C.; Liu, X.; Fang, P.; Tong, Y.; Lu, X. Adv. Mater. 2019, 31, 1904948.

[33] Zhang, N.; Cheng, F.; Liu, Y.; Zhao, Q.; Lei, K.; Chen, C.; Liu, X.; Chen, J. J. Am. Chem. Soc. 2016, 138, 12894.

[34] Liu, C.; Neale, Z.; Zheng, J.; Jia, X.; Huang, J.; Yan, M.; Tian, M.; Wang, M.; Yang, J.; Cao, G. Energy Environ. Sci. 2019, 12, 2273.

[35] Ma, L.; Chen, S.; Li, H.; Ruan, Z.; Tang, Z.; Liu, Z.; Wang, Z.; Huang, Y.; Pei, Z.; Zapiena, J.; Zhi, C. Energy Environ. Sci. 2018 
11,2521

[36] Huang, J.; Wang, Z.; Hou, M.; Dong, X.; Liu, Y.; Wang, Y.; Xia, Y. Nat. Commun. 2018, 9, 1 .

[37] Zhang, N.; Cheng, F.; Liu, J.; Wang, L.; Long, X.; Liu, X.; Li, F.; Chen, J. Nat. Commun. 2017, $8,1$.

[38] Liu, J.; Wang, J.; Ku, Z.; Wang, H.; Chen, S.; Zhang, L.; Lin, J.; Shen, Z. ACS nano, 2016, 10, 1007.

[39] Liu, J.; Chen, M.; Zhang, L.; Jiang, J.; Yan, J.; Huang, Y.; Lin, J.;
Fan, H.; Shen, Z. Nano Lett. 2014, 14, 7180.

[40] Dai, X.; Wan, F.; Zhang, L.; Cao, H.; Niu, Z. Energy Storage Mater. 2019, 17, 143 .

[41] Alfaruqi, M.; Gim, J.; Kim, S.; Song, J.; Pham, D.; Jo, J.; Xiu, Z.; Mathew, V.; Kim, J. Electrochem. Commun. 2015, 60, 121.

(Cheng, B.) 